SISTEMA
ELETROANICO
DE REVISTAS
SER I UfPR

\title{
Produtividade agrícola e mudanças socioculturais: a agricultura quilombola no Vale do Ribeira-SP Brasil
}

\section{Agricultural productivity and socio-cultural changes: quilombola agriculture in Vale do Ribeira-SP Brazil}

\author{
Daniela IANOVALI ${ }^{1 *}$, Cristina ADAMS ${ }^{1}$, Alexandre Antunes RIBEIRO FILHO ${ }^{1}$, Carlos Armênio \\ KHATOUNIAN $^{1}$ \\ ${ }^{1}$ Universidade de São Paulo (USP), São Paulo, SP, Brasil. \\ *E-mail de contato: danianovali@gmail.com
}

Artigo recebido em 5 de setembro de 2017, versão final aceita em 24 de agosto de 2018.

RESUMO: O sistema agrícola itinerante (SAI), uma das formas mais antigas de agricultura, ainda é praticado pelas comunidades quilombolas do Vale do Ribeira-SP, mesmo que venha sendo parcialmente substituído pela agricultura permanente e comercial em monocultivo de pupunheira para palmito. Este artigo teve como objetivo avaliar a produtividade entre os diferentes sistemas de cultivo, discutindo os motivos e impactos desta transição e sua sustentabilidade, assim como os impactos econômicos para as comunidades. Na metodologia foram utilizados: itinerário técnico, entrevistas semiestruturadas, visitas a campo e cálculo do valor agregado líquido. Com relação à rentabilidade dos diferentes sistemas (itinerante e permanente) a agricultura permanente se mostrou mais eficiente em termos de renda e no uso do trabalho, do que o SAI. Entretanto, o SAI desempenha um papel não só de produção de alimentos, mas também como parte de um complexo de relações socioambientais.

Palavras-chave: agricultura itinerante; comunidade rural; conhecimento tradicional; corte-e-queima.

ABSTRACT: The shifting cultivation system (SCS), one of the oldest forms of agriculture, is still practiced by quilombola communities in the Vale do Ribeira - SP. The production of SCS for domestic consumption is gradually being replaced by permanent and monoculture commercial cultivation of peach palm. This article aimed to assess the productivity of the different cultivation systems and, between areas under different fallow lenghts, discussing the reasons and the impacts of this transition, its sustainability and economic impacts for communities. As for the evaluation of productivity between the two different systems, permanent agriculture was more efficient in 
terms of income and the use of labor than SCS. However, SCS plays a role not only in food production, but it is also part of a complex socio-environmental relations.

Keywords: shifting cultivation; rural community; traditional knowledge; slash-and-burn.

\section{Introdução}

A agricultura praticada por pequenos produtores (smallholders) nas florestas tropicais é caracterizada por uma grande variedade de sistemas. Esta variedade é resultante da alocação individual dos recursos disponíveis aos agricultores (terra, trabalho, tecnologias, água), que é realizada dentro de um determinado ambiente ecológico, cultural e institucional e está sujeita a imprevistos climáticos, de mercado e ocorrências no âmbito da unidade doméstica (Barlett, 1980; Ellis, 2000). Sistemas agrícolas familiares são, portanto, dinâmicos e sujeitos a constantes transformações.

Até meados do século XX, o sistema conhecido por agricultura itinerante (shifting cultivation) foi a principal forma de cultivo nos trópicos, sendo considerada a escolha mais racional sob determinadas condições demográficas (baixa densidade populacional), ambientais (solos pouco férteis) e econômicas (acesso desigual aos mercados, uso de técnicas de baixo impacto) (Fox et al., 2000; Mertz, 2002; Van Vliet et al., 2012; FAO, 2015).

$\mathrm{O}$ sistema agrícola itinerante (SAI) pode ser definido, de forma ampla, como qualquer sistema agrícola contínuo no qual clareiras são abertas na floresta para serem cultivadas por períodos de tempo mais curtos do que aqueles destinados ao pousio, implicando numa rotação das áreas sob cultivo na paisagem (Conklin, 1961; Kleinman et al., 1995).
Contanto que a capacidade suporte do ambiente não seja excedida e o período de pousio seja adequado para a recomposição florestal, o SAI pode ser considerado como uma forma sustentável de agricultura (Adams, 2000a; Jakovac et al., 2015; Ribeiro Filho et al., 2018).

No Brasil, o SAI recebe diversas denominações, como cultivo/agricultura itinerante, roça de coivara, roça de toco, e agricultura de corte-e-queima, normalmente considerados sinônimos entre pesquisadores (Neves et al., 2012). Neste trabalho, diferenciamos o SAI da agricultura de corte-e-queima (slash-and-burn cultivation), que implica na conversão florestal permanente para outros tipos de uso do solo, como áreas de pasto ou cultivo comercial, e que dificultam ou atrasam o processo de regeneração natural, concordando com Van Vliet et al. (2013).

Apesar do SAI ter se transformado ao longo da história (Cramb et al., 2009) e desaparecido de algumas paisagens, continua sendo praticado em diversas regiões do mundo (Van Vliet et al., 2012), sendo considerados sistemas altamente adaptativos, capazes de se transformar e responder a variações populacionais e diferentes graus de incorporação ao mercado (Padoch \& Pinedo-Vasquez, 2010).

$\mathrm{O}$ desenvolvimento e a evolução histórica da agricultura podem ser explicados por modelos que se baseiam na relação entre população e tecnologia, explicando a intensificação da agricultura através da 
produtividade do trabalho e da terra (ver Mazoyer \& Roudart, 2010). Uma das variáveis explicativas centrais às teorias de intensificação agrícola é a produtividade do trabalho (Boserup, 1987; Stone, 2001; Nielsen et al., 2006). Durante as décadas de 1970-80, a antropologia deu a sua contribuição a este debate através da investigação dos aspectos produtivos de diversos sistemas agrícolas tropicais (Barlett, 1980; Turner \& Brush, 1987), incluindo alguns praticados na floresta Amazônica (Denevan, 1971; Carneiro, 1983; Vickers, 1983). Todavia, poucos estudos tratam sobre o tema da produtividade de sistemas agrícolas itinerantes no bioma da mata atlântica (Ianovali, 2015).

\subsection{O sistema agrícola itinerante}

Os sistemas agrícolas itinerantes são caracterizados basicamente por três fases: (1) conversão, (2) cultivo e (3) pousio (Kleinman et al., 1995). A conversão consiste na derrubada e queima da vegetação florestal (normalmente secundária); o período de cultivo varia dependendo da região, mas normalmente é de 1 a 3 anos; o pousio pode ser natural ou manejado e tem a função de recuperar as condições do solo antes da derrubada. Sua duração também é variável, mas suficientemente longa para que a vegetação lenhosa se torne dominante (Kleinman et al., 1995; Mertz et al., 2009). A maior demanda

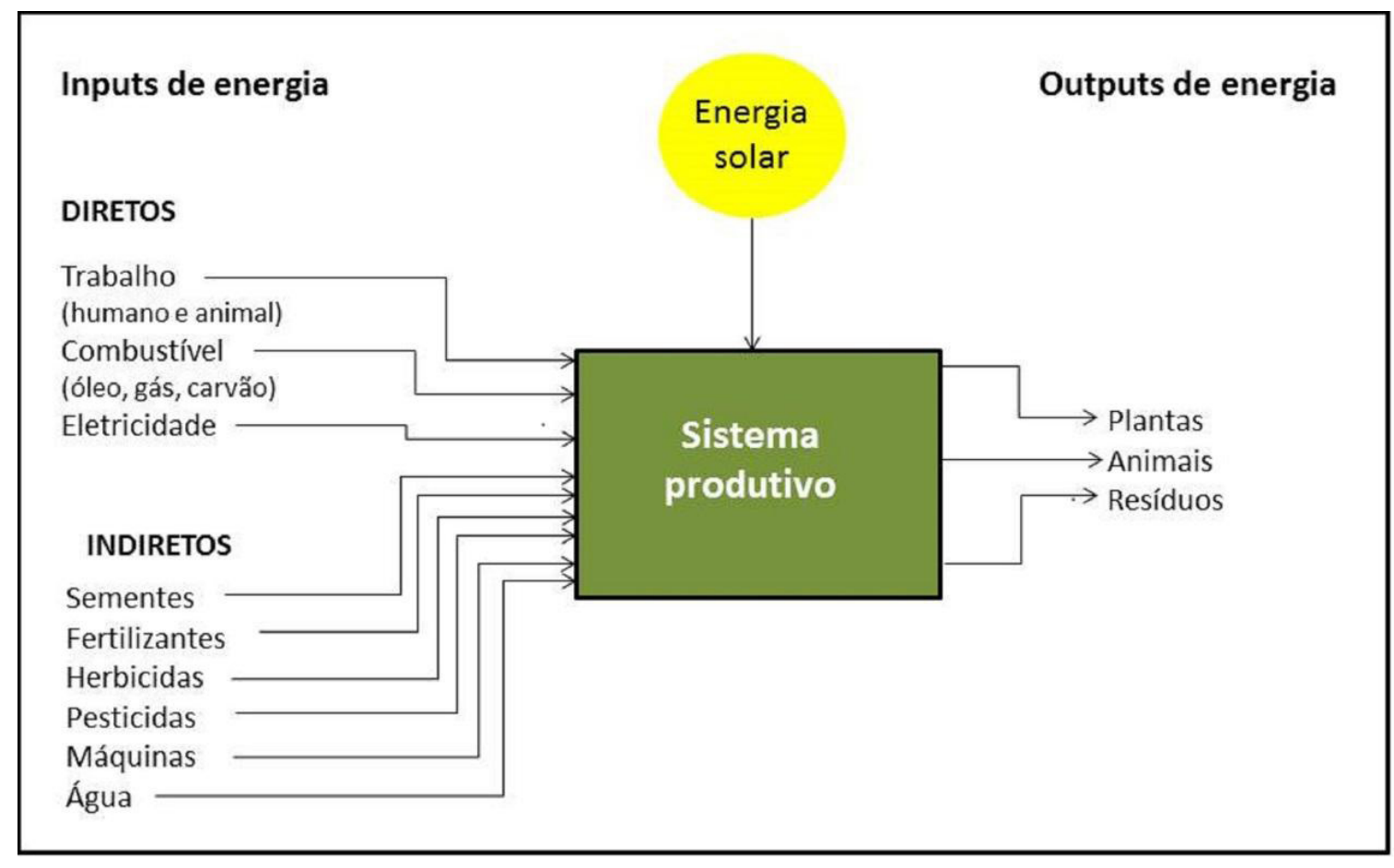

FIGURA 1 - Esquema de inputs e outputs de energia num sistema agrícola

FONTE:<https://www.e-education.psu.edu/geog030/book/export/html/134>. Acesso em: 01 mar. 2014. 
de trabalho ocorre na derrubada da vegetação, que normalmente é realizada em sistema de mutirão (Pedroso Junior, 2008; Pedroso Junior et al., 2008).

A razão do sucesso e da ubiquidade do SAI nas florestas tropicais é atribuída ao fato do sistema aproveitar as características ecológicas dos solos pouco férteis e os nutrientes acumulados na biomassa (Adams, 2000b; Ribeiro Filho, 2015), e à sua capacidade de produzir uma grande variedade de cultivares (agrobiodiversidade) com baixa utilização de energia (trabalho) e tecnologia (Boserup, 1987; Whitmore, 1998, Padoch \& Pinedo-Vasquez, 2010), contribuindo para a segurança alimentar das unidades domésticas.

Todavia, há poucos trabalhos detalhados sobre a produtividade de SAIs, dada a notória dificuldade em coletar dados sobre investimento de trabalho em sistemas agrícolas de pequenos produtores, e o fato de muitos destes sistemas terem se modernizado e utilizarem insumos químicos que substituem o trabalho (Nielsen et al., 2006). Além disso, muitos dos sistemas de economia familiar, que dependiam exclusivamente da agricultura itinerante, passaram a incorporar outros sistemas produtivos e também o trabalho não-agrícola externo à propriedade ou unidade doméstica, como é o caso estudado por Nielsen et al. (2006) e do SAI investigado aqui (Adams et al., 2013).

De forma geral, o cálculo da produtividade de qualquer sistema agrícola implica numa avaliação do conjunto de insumos (como terra, trabalho, capital, fertilizantes e defensivos), que conduz à obtenção de um determinado conjunto de produtos, permitindo descrever os processos pelos quais os produtos agrícolas são produzidos (IEA, 1986), como mostra a Figura 1. Uma análise da relação produtos:insumos ou output:input feita por Bayliss-
-Smith (1982) mostra que o uso eficiente da energia nos sistemas agrícolas diminui conforme o grau de dependência de energias fósseis aumenta.

Quanto ao investimento em trabalho, Beckerman (1987) compilou informações sobre sistemas indígenas em florestas tropicais da América do Sul, ainda que não houvesse dados coletados para todas as etapas do SAI. A variação encontrada (ilustrada na Tabela 1) pode ter causas nos diferentes tipos de cultivos e no investimento do tempo em outras atividades, como a caça e a coleta.

Outra característica importante dos SAIs, no que se refere ao investimento em trabalho, é que a demanda por mão-de-obra da unidade doméstica é sazonal, liberando o agricultor para realizar outras atividades complementares (Dove, 1983; Beckerman, 1987). Dove (1983), estudando os Kantu' da Indonésia, mostrou que o SAI permitia disponibilizar entre 100 (em áreas abertas em floresta secundária) e 200 dias/ano/unidade doméstica (áreas em floresta primária) para outras atividades. Esses dias foram utilizados, muitas vezes, para trabalhar nas áreas de outros agricultores da comunidade, que depois retribuíam o período recebido; no Vale do Ribeira este processo é conhecido como troca-dia (Munari, 2009).

A relação entre o investimento em trabalho e a produção agrícola dos SAIs pode ser medida através da produtividade do trabalho, como fizeram Nielsen et al. (2006) em áreas de produção de arroz no sudeste asiático. Dados primários dos autores mostraram que a existência de oportunidades de trabalho remunerado diminui a quantidade total de trabalho investido no SAI e o tamanho das roças, mas não influencia no tempo de trabalho investido por hectare no sistema. A alocação do tempo em diversas atividades não interferiu na produtivida- 
de da agricultura itinerante, que foi considerada relativamente alta $(11,0 \mathrm{~kg}$ de arroz com casca por homem-dia, no mínimo) e competitiva economicamente com as outras oportunidades de trabalho fora da produção familiar.

Uma das críticas feitas aos trabalhos que buscaram calcular o input energético do trabalho e o output em termos de produção alimentar é que eles não consideraram a contribuição energética da vegetação derrubada e queimada para a abertura da roça (McGrath, 1987). Para McGrath (1987), o SAI só possui alta produtividade (20:1, output:input) quando o valor energético da biomassa presente na floresta derrubada não é incluído na equação, o que resultaria numa relação de 0,11:1.

A importância da biomassa na produção de alimentos é reconhecida por agricultores itinerantes, estando relacionada ao tempo de pousio das áreas onde é implantada uma nova roça. Teoricamente, mantidas as demais variáveis constantes, quanto maior o tempo de pousio, maior será a produtividade do SAI (Mertz, 2002). Entretanto, conforme Mertz (2002), nem sempre esta relação se verifica, desmistificando a suposição de que pousios mais curtos levariam necessariamente a um colapso do sistema. A utilização de períodos mais curtos poderia, por exemplo, estar relacionada a um aumento do custo de oportunidade do agricultor, que prefere utilizar áreas mais próximas à sua casa e, talvez, investir no uso de insumos químicos (Nielsen et al., 2006).

\subsection{O SAI Quilombola do Vale do Ribeira em transformação}

A agricultura itinerante tem sido praticada pelas comunidades quilombolas do Vale do Ribeira desde sua formação entre os séculos XVIII e XIX.

TABELA 1 - Investimento em trabalho (homens-hora/ha) em sistemas agrícolas itinerantes de diferentes povos indígenas sul-americanos (adaptado de Beckerman, 1987).

\begin{tabular}{|c|c|c|c|c|c|c|c|}
\hline Tronco linguístico & Povo & Roçada* & Derrubada & Queimada & Plantio & $\begin{array}{l}\text { Colheita/ } \\
\text { replantio }\end{array}$ & Total \\
\hline Macro-Chibchan & Barí & 31 & 55 & 1 & 193 & 570 & 850 \\
\hline Macro-Caribe & Kuikuru & & & 11 & 180 & & 191 \\
\hline Macro-Tucano & Siona-Secoya & 47 & 16 & & 186 & 634 & 883 \\
\hline \multirow{3}{*}{ Arawak } & Campa & \multicolumn{2}{|c|}{70} & & & & 70 \\
\hline & Mirití-Paraná & 32 & 88 & 1 & & & 121 \\
\hline & San Carlos & & 126 & 6 & 166 & 419 & 717 \\
\hline Macro-Panoa & Shipibo & & 91 & & 75 & 176 & 342 \\
\hline
\end{tabular}

* A roçada é a primeira etapa de limpeza da área e envolve o corte de plantas herbáceas e cipós, facilitando o trânsito pela área. O trabalho de capina nestes sistemas (homens-hora/ha/ano) variou entre 75 e 900 (Beckerman, 1987). 
Durante o século XX, a região passou por diversos ciclos econômicos (mineração, produção de chá e banana), que passaram ao largo do SAI quilombola. Fatores como a melhoria de acesso e a maior integração ao mercado, a criação de unidades de conservação na região e as modificações na legislação ambiental, influenciaram a prática da agricultura itinerante pelas comunidades quilombolas (Adams et al., 2013; Futemma et al., 2015).

Tais mudanças sociais e econômicas, aliadas ao aumento da escolaridade média e à possibilidade de prestação de serviços em fazendas da região, contribuíram para a diminuição desta atividade agrícola tradicional de produção de alimentos para consumo doméstico e para a diversificação do SAI quilombola, fenômeno também observado em outras regiões (Vicente \& Fantini, 2014; Schwartz \& Corzo, 2015; Wood et al., 2016).

De acordo com as leis brasileiras, a roça só é permitida mediante licença ambiental em áreas em estágio inicial de sucessão, conforme a Lei da Mata Atlântica (Lei Federal 11.428/2006 e Decreto Federal 6.600/2008), o que contraria o conhecimento tradicional local, que indica áreas em estágios mais avançados como as ideais para plantio (Brasil, 2006; 2008).

Outra consequência destas mudanças histórico-culturais foi a adoção de novos cultivares comerciais. A partir dos anos 2000, os agricultores quilombolas aderiram ao cultivo da pupunheira (Bactris gasipaes Kunth) para a produção de palmito como opção à extração do palmito nativo juçara (Euterpe edulis Mart.) e ao cultivo comercial de banana, já consolidado na região. Esta nova fonte de renda é apoiada por órgãos de assistência técnica e pesquisa da região do Vale do Ribeira, e por incentivos de políticas públicas de crédito agrícola (Ianovali, 2015).

\subsection{Agricultura familiar e meios de vida.}

As populações tradicionais brasileiras que herdaram a agricultura itinerante das sociedades indígenas que ocupavam as florestas amazônica e atlântica podem ser consideradas, de uma forma geral, como agricultores familiares. A perspectiva mais recente sobre a agricultura familiar reconhece suas múltiplas funções, relativizando o viés desenvolvimentista baseado em intensa tecnificação e aumento da produtividade (Khatounian, 2001; Mazoyer \& Roudart, 2010; Gavioli \& Costa, 2011).

A agricultura itinerante tem sido utilizada pelas comunidades Quilombolas do Vale do Ribeira não só como uma estratégia produtiva ou tradicional, mas também como parte de seus bens culturais materiais e imateriais. Levantamento recente (Andrade \& Tatto, 2013) demonstrou a centralidade do sistema agrícola baseado na roça com relação à identidade quilombola. O "modo de fazer roça" está ligado a diversos outros bens culturais das comunidades como formas de expressão e celebrações.

Conforme a abordagem de livelihoods (Ellis, 2000), o termo "meio de vida" se refere não apenas ao que as pessoas fazem para ganharem a vida ou sobreviverem, mas também aos recursos que possibilitam a capacidade de criar uma vida satisfatória; inclui, também, o risco que precisa ser considerado no manejo dos recursos; e o contexto social e institucional no qual a unidade doméstica está inserida.

Para Ellis (2000), os meios de vida podem ser vistos como o acesso a cinco diferentes tipos de capitais: capital humano (saúde, nutrição, educação, conhecimentos e habilidades, capacidade de trabalhar e capacidade de se adaptar); capital natural (terra e produção, recursos hídricos, árvores e florestas, biodiversidade, serviços ecossistêmicos); capital 
social (redes e conexões, relações de confiança e reciprocidade, grupos formais e informais); capital físico (infraestrutura, ferramentas e tecnologias); e capital financeiro (estoques, créditos, poupanças).

Para Luca \& Kubo (2015), meios de vida sustentáveis são meios de vida que possibilitam aumentar as capacitações das pessoas, fazendo-as mais resistentes a crises e a mudanças, tornando-as mais resilientes. Este conceito dialoga com o conceito de capital cultural (Bebbington, 1999) que envolve as qualidades simbólicas que se referem às visões de mundo dos agricultores.

Embora nem todos os parâmetros relativos aos meios de vida tenham sido coletados de forma sistemática durante a pesquisa, as informações sobre a unidade doméstica e seu meio de vida foram utilizados para discutir os resultados.

Neste artigo, comparamos a produtividade do trabalho agrícola quilombola (Vale do Ribeira-SP) no SAI e no cultivo comercial da pupunha (Bactris gasipaes Kunth), através de um balanço entre os insumos e produtos em cada um dos sistemas, incluindo variações na biomassa (tempo de pousio). A seguir, no item 2, estão descritos os materiais e métodos, seguidos pelos resultados (item 3) e a discussão (item 4). O item 5 conclui o artigo.

\section{Material e Métodos}

\section{1. Área de estudo}

O Vale do Ribeira (2.830.666 ha) está situado entre as cidades de São Paulo e Curitiba e mantém a maior área remanescente de mata atlântica do Brasil. A região abrange 21 comunidades remanescentes de quilombos, das 28 encontradas no Estado de São Paulo (Andrade \& Tatto, 2013).

A formação destas comunidades quilombolas está ligada à atividade de mineração na região entre os séculos XVII e XVIII (ITESP, 2003). Com o declínio da atividade que migrou para o Estado de Minas Gerais, a mão de obra negra escrava passou a ser menos requerida, possibilitando que homens e mulheres escravos se estabelecessem como camponeses autônomos em comunidades ao longo do Rio Ribeira (Queiroz, 1983; ITESP, 2000; 2003).

A pesquisa foi realizada nos Quilombos de São Pedro e Pedro Cubas de Cima, ambos localizados no município de Eldorado-SP (Figura 2).

\subsection{Delineamento}

A pesquisa foi concebida como um estudo de caso descritivo, que teve o objetivo de levantar dados sobre a produtividade do trabalho no sistema agrícola tradicional Quilombola e no cultivo comercial da pupunha (Bactris gasipaes Kunth), que vem sendo proposto como alternativa para geração de renda nas comunidades. A unidade de análise foi a unidade doméstica (UD), definida como a unidade básica de produção e reprodução socioeconômica (Netting, 1993; Bernard, 2005), e sua área agrícola (uma por unidade doméstica).

Dada a importância reportada pelos Quilombolas e pela literatura sobre o tempo de pousio para o sucesso do SAI (Carriere et al., 2002; Ribeiro Filho, 2015), foram estabelecidas inicialmente duas categorias de idade para as florestas secundárias (capoeiras) derrubadas para o plantio: em torno de 10 anos, conforme o limite legal para a derrubada de vegetação secundária, e entre 20-25 anos, que é o 
tempo considerado necessário para a recuperação do solo segundo os agricultores quilombolas. Durante a escolha das áreas, o tempo de pousio foi informado pelos agricultores.

A escolha das áreas agrícolas beneficiou-se do delineamento amostral de Ribeiro Filho (2015), que avaliou o impacto do SAI sobre os solos. Todas elas foram pré-definidas de comum acordo com as comunidades locais, e licenças de pesquisa foram obtidas junto ao órgão ambiental responsável (CETESB/SMA-SP) para a derrubada e a queima da vegetação. $\mathrm{O}$ delineamento amostral inicialmente proposto incluía 13 áreas (7 com 10-15 anos de pousio e 6 com 20-25 anos), sendo 6 em São Pedro (SP) e 7 em Pedro Cubas de Cima (PCC). Para o cultivo de pupunheira (Bactris gasipaes Kunth) foram estudadas 5 áreas com período de implantação variando de 1 a 4 anos, sendo 3 em SP e 2 em PCC. A dimensão média das roças foi de 0,4 ha, e no caso do cultivo da pupunha de 0,5 ha.

\subsection{Coleta de dados}

Entrevistas estruturadas e semiestruturadas foram realizadas durante a execução da pesquisa

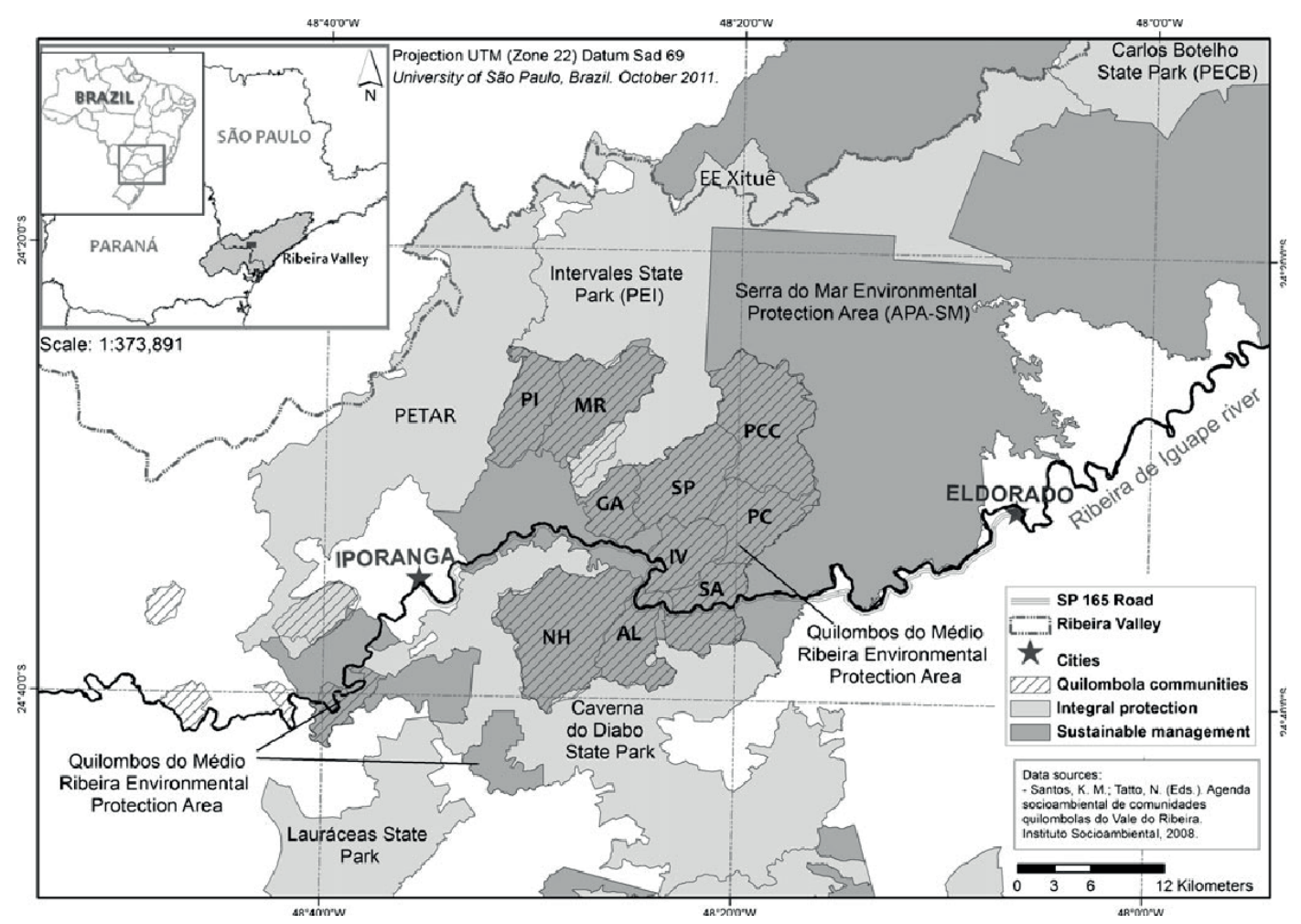

FIGURA 2 - Comunidades quilombolas e unidades de conservação.

FONTE: Adams et al. (2013). 
em diferentes períodos da safra. Para o estudo do SAI, as entrevistas foram complementadas com observação participante (Raupp \& Beuren, 2006), e as fases de derrubada da mata, roçada, queimada, plantio e colheita foram acompanhadas (agosto de 2013 a julho de 2014). Para o estudo do cultivo permanente de pupunha foram realizadas as entrevistas e visitas a campo. Foram observadas as práticas agrícolas, as tecnologias utilizadas, o tempo destinado ao trabalho e o "modo de fazer", além de aspectos culturais da UD e para cada uma das áreas agrícolas foi realizada a reconstituição do itinerário técnico (Dufumier, 2010). O trabalho também se beneficiou de informações coletadas em pesquisas anteriores realizadas com as mesmas comunidades (Pedroso Junior, 2008; Munari, 2009).

\subsection{Análise de dados}

Para avaliar a produtividade dos dois sistemas foram levantados os seguintes parâmetros:

- entradas (inputs): tempo destinado ao trabalho, ferramentas utilizadas, quantidade de sementes e mudas plantadas, área destinada ao cultivo e insumos utilizados. Foram mensurados os custos com insumos, ferramentas, mudas e sementes, da mão de obra e do combustível utilizado;

- saídas (outputs): a quantidade produzida (em quilos por hectare) e o rendimento monetário proporcional às vendas no mercado local.

A quantificação do input de biomassa das áreas em diferentes estágios de regeneração da vegetação foi realizada através da estimativa do peso seco (InPS), anteriormente à fase de derrubada, com modelos que utilizam o diâmetro da base (d) e a altura (h) dos indivíduos arbóreos acima de 1,5 metros de altura (Barbosa et al. 2014, Ribeiro Filho, 2015). Os modelos utilizados para o cálculo de biomassa foram desenvolvidos por Burger (2005) através de método destrutivo, e são descritos pelas Fórmulas (1) e (2):

Modelo 1: $\ln P S(k g)=-4,15190+1,06068 \ln (\mathrm{d} 2 \mathrm{~h})$ Modelo 2: $\ln P S(k g)=-6,71710+1,30308 \ln (\mathrm{d} 2 \mathrm{~h})$ (2)

Sendo: peso seco (InPS), diâmetro da base (d) e a altura (h) de indivíduos arbóreos acima de 1,5 metros de altura.

A produtividade agrícola bruta foi mensurada através da relação hora-homem e quilos por hectare $(\mathrm{H}-\mathrm{H} / \mathrm{ha}$ e $\mathrm{kg} / \mathrm{ha}$ respectivamente) (Boserup, 1987; Turner \& Brush, 1987; Nielsen et al. 2006; Mazoyer \& Roudart, 2010). As horas de trabalho foram contabilizadas para a área real de cultivo e depois extrapoladas para um hectare, para terem a mesma base de comparação. Para o cálculo dos resultados econômicos foi utilizada a relação entre a produção e os custos anuais no cálculo do valor agregado líquido (VAL), de acordo com a Fórmula (3) (adaptada de Dufumier, 2010):

$$
\mathrm{VAL}=\mathrm{PB}-\mathrm{Cl}
$$

Sendo: VAL: valor agregado líquido; PB: produção bruta anual; $\mathrm{Cl}$ : valor do consumo intermediário anual, ou total dos custos o total de custos (insumos, investimentos, sementes, equipamentos). 


\section{Resultados}

A roça (SAI) envolveu as seguintes etapas:

1. Roçada da vegetação, com uso de foice para a retirada da vegetação mais fina, como arbustos, capim e cipós;

2. Derrubadas árvores de maior porte e corte (o "picar") dos troncos e galhos, feito com motosserra ou machados;

3. Queimada da matéria orgânica, que ocorreu aproximadamente 20 dias após a derrubada. Esta etapa demonstrou muitas nuances de conhecimento entre os diferentes agricultores, mas no geral foi realizada no horário mais quente do dia, após cerca de 20 dias sem chuva e com vento a favor;

4. Plantio, realizado com equipamentos como matraca ou vara de plantar;
5. Colheita de milho e arroz, sendo utilizado o canivete para o corte dos cachos de arroz.

O tempo despendido em cada uma delas pode ser visto na Tabela 2. Das 13 áreas inicialmente propostas para este trabalho, apenas seis realizaram as etapas de preparo (1-3), cinco receberam sementes e três (em negrito na Tabela 2 ) chegaram ao estágio de produção. A falta de mão de obra foi o fator desencadeador da desistência de alguns agricultores, além de problemas com a titularidade e posse da terra. Fatores climáticos também influenciaram o abandono da pesquisa por parte de alguns agricultores. A seca ocorrida em janeiro de 2014 prejudicou a cultura do milho e inviabilizou a colheita em uma das unidades amostrais. Como, devido à seca, a produção de milho e arroz nas roças foi suficiente apenas para que os agricultores não perdessem as

TABELA 2- Exigência de trabalho (hora-homem por hectare, H-H/ha) para preparo e cultivo de roça relacionado à idade da capoeira.

\begin{tabular}{|c|c|c|c|c|c|c|c|c|c|c|c|}
\hline \multirow{2}{*}{ Capoeira } & \multirow{2}{*}{ Código } & \multirow{2}{*}{$\begin{array}{l}\text { Pousio } \\
\text { (anos) }\end{array}$} & \multicolumn{2}{|c|}{ Biomassa (t/ha) } & \multirow{2}{*}{$\begin{array}{l}\text { Roçar } \\
\text { H-H/ha }\end{array}$} & \multirow{2}{*}{$\begin{array}{c}\text { Derrubar } \\
\text { H-H/ha }\end{array}$} & \multirow{2}{*}{$\begin{array}{c}\text { Picar } \\
\text { H-H/ } \\
\text { ha }\end{array}$} & \multirow{2}{*}{$\begin{array}{l}\text { Queimar } \\
\text { H-H/ha }\end{array}$} & \multirow{2}{*}{$\begin{array}{l}\text { Plantar } \\
\text { H-H/ha }\end{array}$} & \multirow{2}{*}{$\begin{array}{l}\text { Total } \\
\text { H-H/ha }\end{array}$} & \multirow{2}{*}{$\begin{array}{l}\text { Total } \\
\text { H-H/ } \\
\text { ha.dic }\end{array}$} \\
\hline & & & Mod. 1 & Mod. 2 & & & & & & & \\
\hline \multirow[t]{4}{*}{ Jovem } & PCC6 & 10 & 30,18 & 13,62 & 37 & 10 & 11 & 0,5 & 53 & 113 & 12 \\
\hline & SP5 & 12 & 45,13 & 20,27 & 44 & 32 & 11 & 02 & 103 & 195 & 24 \\
\hline & SP2 & 15 & 114,39 & 72,72 & 35 & 23 & 04 & 03 & 78 & 145 & 18 \\
\hline & & Média & & & 39 & 22 & 09 & 02 & 78 & 151 & 18 \\
\hline \multirow[t]{4}{*}{ Avançada } & $\mathrm{PCC} 7$ & 25 & 201,74 & 149,10 & 33 & 68 & 35 & 01 & 122 & 261 & 35 \\
\hline & SP6 & $25-30$ & 336,80 & 252,14 & 28 & 56 & - & 10 & - & 96 & 12 \\
\hline & PCC2 & 30 & 226,01 & 169,93 & 22 & 65 & 21 & 02 & 34 & 147 & 18 \\
\hline & & Média & & & 28 & 63 & 28 & 04 & 78 & 204* & $26 *$ \\
\hline
\end{tabular}

Nas áreas PCC6 e PCC7 foi usado apenas o machado para a derrubada; na área SP5 foi usada motosserra; e nas demais áreas foi usada uma combinação de machado e motosserra. Biomassa calculada por Barbosa et al. (2014) e Ribeiro Filho (2015). O dia de trabalho foi calculado como sendo de 8 horas.

*Nota: as médias de trabalho em 204:01* horas e em 26* dias foram calculadas sem o valor da área SP6, pois esta área não houve a etapa de plantio 
sementes (13-88 $\mathrm{kg}$ de milho e $84 \mathrm{~kg}$ de arroz), optou-se por substituí-la pela expectativa de produção dos agricultores, conforme a experiência de anos anteriores. A produtividade esperada do milho variou entre $660-1.600 \mathrm{~kg} / \mathrm{ha}$, com uma média de $980 \pm 354 \mathrm{~kg} / \mathrm{ha}$. Já para o arroz, a produtividade esperada variou entre $620-2.400 \mathrm{~kg} / \mathrm{ha}$, com uma média de $1.395 \pm 640 \mathrm{~kg} / \mathrm{ha}$. Desta forma, não foi possível avaliar a relação entre o input de biomassa e a produção agrícola do SAI Quilombola.

As áreas de cultivo da pupunheira localizavam-se mais próximo das residências. O plantio foi feito numa área já aberta, sem necessidade de suprimir a vegetação. A limpeza do terreno foi feita com capina e, às vezes, fogo. A exigência de trabalho para o cultivo da pupunheira (dia/homem por hectare) foi calculada desde o preparo da área até a colheita (Tabela 3). Para este levantamento foram realizadas entrevistas semiestruturadas baseadas em uma planilha de atividades e orientações técnicas fornecidas pela CATI - Coordenadoria de Assistência Técnica Integral de Registro para indicações técnicas ao cultivo da pupunheira para palmito.

Para possibilitar a comparação entre os dois sistemas quanto a seus insumos e produtos, calcu- lou-se o valor agregado líquido para cada uma das áreas (Tabela 4). Para o cálculo do valor da hora de trabalho no SAI utilizamos os valores de diárias locais para serviços braçais, que variou entre $\mathrm{R} \$ 35$ e R \$40. Os valores de venda da pupunha e do milho foram obtidos em agosto de 2013, no mercado local do município de Eldorado-SP: R \$50,00 um saco de milho de $60 \mathrm{~kg}$ e $\mathrm{R} \$ 3,00^{1}$ uma haste de palmito de pupunha. Assim, para efeito do cálculo do valor agregado líquido (VAL), ou dos resultados econômicos dos dois sistemas, estes foram os valores considerados (Dufumier, 2010).

Na primeira parte da Tabela 4 verifica-se que o VAL, ou a remuneração pelo dia de trabalho no SAI descontados os custos de produção, variou de $\mathrm{R} \$ 22,00$ a R $\$ 34,00 /$ dia. Já no cultivo de pupunheira, o VAL variou entre $\mathrm{R} \$ 48,00$ e $\mathrm{R} \$ 168,00 /$ dia.

$\mathrm{Na}$ impossibilidade de levantar os dados de produção, foi estimada junto aos agricultores a quantidade de arroz e milho que poderiam ser produzidos, de acordo com a memória de cada agricultor quilombola. Nesta etapa, foram avaliadas as expectativas de produção para 10 áreas. A produtividade média esperada para o cultivo do arroz foi de $1.395 \mathrm{~kg} / \mathrm{ha}$ e de milho foi de $981 \mathrm{~kg} / \mathrm{ha}$.

TABELA 3-Dias de trabalho (8h) por agricultor, para tratos da pupunha para palmito, incluindo plantio, manutenção e cortes anuais, por hectare. Fonte: dados coletados pelos autores.

\begin{tabular}{lcccccccccc}
\hline \multirow{2}{*}{ Código } & Ano 1 & Ano 2 & Ano 3 & Ano 4 & Ano 5 & Ano 6 & Ano 7 & Ano 8 & Ano 9 & Ano 10 \\
\cline { 2 - 9 } & (dias) & (dias) & (dias) & (dias) & (dias) & (dias) & (dias) & (dias) & (dias) & (dias) \\
\hline SP5 & 73 & 59 & 59 & 41 & 41 & 41 & 41 & 41 & 41 & 41 \\
SP1 & 62 & 52 & 52 & 52 & 56 & 52 & 52 & 52 & 52 & 103 \\
SP2 & 140 & 103 & 103 & 103 & 103 & 103 & 103 & 103 & 103 \\
PCC1 & 136 & 92 & 92 & 92 & 92 & 92 & 92 & 92 & 92 \\
PCC2 & 190 & 190 & 190 & 190 & 190 & 190 & 190 & 190 & 190 & 190 \\
\hline
\end{tabular}

${ }^{1}$ Em agosto de 2013 o dólar era cotado em R $\$ 2,30$. Portanto o valor da saca de milho é de US\$21,73 e a haste de palmito de pupunha US\$1,30. 
TABELA 4 - Valor agregado líquido (VAL) por hectare para os cultivos do SAI e de pupunheira para palmito. Fonte: dados coletados pelos autores.

SAI

Cultivo permanente

\begin{tabular}{|c|c|c|c|c|c|}
\hline UD & $\begin{array}{c}\text { Dias de trabalho por } \\
\text { hectare }\end{array}$ & $\begin{array}{l}\text { RS por dia por } \\
\text { hectare }\end{array}$ & UD & $\begin{array}{c}\text { Dias de trabalho por } \\
\text { hectare }\end{array}$ & $\begin{array}{l}\text { R\$ por dia por } \\
\text { hectare }\end{array}$ \\
\hline SP5 & 31 & 22 & SP5 & 48 & 68 \\
\hline PCC2 & 20 & 34 & $\mathrm{PCC} 2$ & 190 & 48 \\
\hline \multirow[t]{2}{*}{ Média } & 23,3 & 30 & SP1 & 53 & 65 \\
\hline & & & Média & 98,8 & 89 \\
\hline
\end{tabular}

\section{Discussão}

\subsection{Relações de input x output - Recursos} financeiros e fatores de risco

A redução na amostra de áreas agrícolas investigadas reflete a dificuldade em se levantar informações sobre a produtividade em SAIs como apontado por Nielsen et al. (2006). Tanto os fatores climáticos, como as mudanças socioculturais (Luca $\&$ Kubo, 2015) pelas quais passam as comunidades quilombolas (como a redução da mão-de-obra disponível na unidade doméstica), influenciaram no abandono da área agrícola por parte dos agricultores.

Nas áreas que foram cultivadas, o número médio de horas de trabalho investido pelos quilombolas no SAI variou entre 96 e $261 \mathrm{H}-\mathrm{H} / \mathrm{ha}$ (Tabela 2), encontrando-se dentro da faixa levantada por Beckerman (1987) em outros sistemas, que variou de 92 a $280 \mathrm{H}-\mathrm{H} / \mathrm{h}$ da roçada até a queimada (excetuando-se o plantio e a colheita). Nielsen et al. (2006) estimaram o investimento de trabalho para os sistemas do sudeste asiático em 117 dias/homem por hectare.

Embora em campo os agricultores quilombolas tenham afirmado que é mais difícil trabalhar numa área de capoeira jovem devido à presença de capim e herbáceas, nossos resultados mostram o contrário.

Nas áreas quilombolas com pousio de 10-15 anos, o investimento médio em $\mathrm{H}-\mathrm{H} /$ ha foi de $151 \pm 41$, ao passo que nas áreas com floresta em estágio mais avançado de sucessão a média foi de $204 \pm 12$. Em áreas de capoeira avançada (PCC2, PCC7 e SP6) houve grande investimento de tempo de trabalho na derrubada da biomassa, chegando a $63 \mathrm{H}-\mathrm{H} / \mathrm{h}$, uma vez que as árvores possuíam maior diâmetro e altura. Em capoeiras jovens (PCC6, SP2, SP5), o tempo dedicado a derrubada foi de $22 \mathrm{H}-\mathrm{H} /$ ha. Além disto, a atividade de picar os galhos nas áreas de pousio avançado consumiu $28 \mathrm{H}-\mathrm{H} / \mathrm{ha}$, enquanto nas áreas mais jovem este tempo foi de $9 \mathrm{H}-\mathrm{H} / \mathrm{ha}$.

Outra variável relatada pelos agricultores quilombolas é de que o uso do fogo diminui o tempo de trabalho de roçada e capina, o que foi verificado por Norgrove \& Hauser (2015) no Congo, onde áreas 
manejadas sem o uso do fogo tiveram um aumento de $50 \%$ no trabalho investido, mas com aumentos nos ganhos monetários.

Ainda com relação ao tempo de trabalho investido, no SAI quilombola foi possível perceber que outras variáveis influenciaram no input de trabalho para a conversão das áreas de floresta em roças, como a declividade da área, a tecnologia utilizada, a idade dos agricultores, a experiência na realização das atividades, o juízo de valor que cada agricultor tem para as etapas de seu trabalho e as condições climáticas.

$\mathrm{Na}$ área PCC7 na Tabela 2, por exemplo, numa capoeira de 25 anos foram observados os maiores valores para o investimento em trabalho. Nela não houve uso de motosserra para aperfeiçoar o tempo de derrubada e os agricultores mais jovens desta família não sabiam plantar o milho ou arroz de acordo com a técnica tradicional com uso de soquete ou vara de plantar, o que pode ter aumentado a relação hora-homem de trabalho por hectare. Já em SP6, uma capoeira de 25-30 anos, o tempo de trabalho foi menor porque não houve a atividade de picar os galhos; em compensação, seu tempo de queimada foi maior pela dificuldade do fogo se espalhar pela área.

Em outros SAIs, observa-se que a alocação de tempo de trabalho entre a agricultura e outras atividades é muito dinâmica (Nielsen et al. 2006). Nielsen et al. (2006) sugerem que oportunidades de trabalho fora da propriedade rural, sejam eles agrícolas ou não-agrícolas, influenciam o input de trabalho no cultivo itinerante e o total de área cultivada, embora isso não necessariamente signifique menor tempo gasto por hectare.

Para o cultivo permanente de pupunha, o investimento em trabalho encontrado para o primeiro ano de implantação foi de 62 a 190 dias-homem por hectare (Tabela 3). Nos cultivos de pupunha pesquisados por Nishikawa et al. (1998) foram necessários cerca de 84 dias-homem por hectare de trabalho no período de implantação e cerca de 64 dias-homem por hectare para o trabalho de manutenção. Comparando-se o trabalho investido pelos quilombolas na roça (18 a 26 dias-homem/ha, dependendo da idade do pousio) e no cultivo da pupunha, parece que o modelo de Boserup se confirma neste caso. Ou seja, que sistemas mais intensivos (pupunha) demandam um maior investimento em trabalho, conforme discutido por Nielsen et al. (2006). De modo geral, as culturas comerciais melhoram os recursos financeiros da unidade doméstica, mas a especialização para o mercado pode aumentar sua vulnerabilidade (Cramb et al., 2009). Todavia, o pequeno tamanho de nossa amostra indica que esta ainda é uma hipótese a ser testada.

A produtividade média de arroz esperada pelos agricultores, baseado na experiência passada, resultou em $1.263 \mathrm{~kg}$ de arroz por hectare. Este valor está acima daquele encontrado por Nielsen et al. (2006) (1.107 kg/hectare) e Dove (1983) (500 $\mathrm{kg} / \mathrm{ha}$ ). Quanto ao milho, a produtividade esperada estimada pelos Quilombolas é de $981 \pm 388 \mathrm{~kg} /$ ha por hectare, comparável a aquela observada na Guatemala por Schwartz e Cozo (2015), de cerca de $1.293 \mathrm{~kg}$ milho/ha. Segundo os autores, os agricultores guatemaltecos alimentavam uma família de 5 a 6 pessoas com 0,5 ha, contando com os recursos dos quintais domésticos. Para Dove (1983), cada agricultor necessitava de $220 \mathrm{~kg}$ arroz/ano.

Quando os custos de produção são incluídos e o VAL médio dos dois sistemas quilombolas é comparado, verifica-se que a remuneração média do SAI foi cerca de 3 vezes menor do que a da pupunha 
por hectare, mas, por outro lado, o investimento em trabalho foi cerca de 4 vezes menor. O resultado econômico do SAI por dia-hectare foi também inferior à diária paga para serviços braçais na região, que variou entre $\mathrm{R} \$ 35,00 /$ dia e $\mathrm{R} \$ 40,00 /$ dia dependendo da comunidade, mas não substancialmente. Em duas das unidades domésticas investigadas, a remuneração foi equivalente ao limite inferior das diárias braçais $(\mathrm{R} \$ 34,00$ e $\mathrm{R} \$ 35,00)$.

Embora a agricultura itinerante tenha sido considerada primitiva e de baixa produtividade (Boserup, 1987; McGrath, 1987; Turner \& Brush; 1987; Hunt, 2000), os resultados avaliados acima mostram o contrário. Situação semelhante foi observada por Nielsen et al. (2006), que sugerem que a persistência do SAI em determinadas localidades pode ter uma racionalidade econômica com maiores resultados, ao contrário do que normalmente se espera.

\subsection{Uma coexistência da roça e do cultivo de mercado?}

A decisão do agricultor quilombola em alocar seu tempo entre o SAI e o cultivo da pupunha não pode ser analisada através de uma simples avaliação do custo de oportunidade (Chayanov, 1986), pois as atividades não são necessariamente excludentes. De acordo com o tempo de trabalho alocado, é possível ao agricultor manter o trabalho na roça (160dias/hectare por ano) e no cultivo comercial de pupunheira (100 dias/hectare por ano).

As atividades podem ser, portanto, complementares (Ellis, 2000).

Na região, observa-se um processo de intensificação agrícola em algumas das comunidades quilombolas (Adams et al. 2013), com a transição do
SAI para cultivos comerciais permanentes (banana, pupunha)que não tem como fator causal a pressão demográfica (Boserup, 1987; Mertz, 2002, Nielsen et al. 2006). Trata-se de um processo de intensificação causado por fatores institucionais (Futemma et al., 2015), sócio-políticos e econômicos (Adams et al., 2013), conforme apontado por Stone (2001). Embora a visão boserupiana possa nos levar a pensar numa substituição gradual do SAI por sistemas mais intensivos e "menos primitivos" (Nielsen et al., 2006), uma análise da agricultura familiar quilombola pode apontar novos caminhos para o desenvolvimento territorial no Vale do Ribeira.

Ao longo da história, a roça esteve relacionada a um modo de vida específico e desempenhava um importante papel na manutenção dos laços sociais. A reciprocidade, representada pelas trocas de dias de trabalho e de produtos entre parentes e vizinhos, garantia a manutenção do tecido social quilombola (Andrade \& Tatto, 2013). Para as comunidades quilombolas, a agricultura é um dos traços socioculturais que fazem parte da identificação de grupo e também é uma das bandeiras da luta pela terra, diretamente ligada à sua identidade cultural.

Portanto, as comunidades quilombolas do Vale do Ribeira encontram-se numa posição privilegiada no que se refere à construção de uma nova ruralidade, vinculando sustentabilidade e desenvolvimento rural, e valorizando a relação entre sociedade e natureza. Ao planejar o uso da terra nos territórios de forma a reconhecer as múltiplas funções da agricultura familiar, vinculando-a a manutenção da paisagem e à preservação do meio ambiente e das tradições culturais e sociais (Camargo \& Oliveira, 2012; Andrade \& Tatto, 2013), o SAI e formas mais comerciais de cultivo podem conviver lado a lado, contribuindo para manter a identidade quilombola. 
Um dos desafios a serem superados e que preocupa lideranças locais é o desinteresse dos quilombolas mais jovens pelo trabalho na roça, os quais preferem trabalhos urbanos ou trabalhar como diaristas em fazendas da região, conforme observado em estudos similares (Luca \& Kubo, 2015) e no Brasil de forma geral (Camarano \& Abramovay, 1999).

\section{Conclus̃̃es}

Com relação à produtividade do trabalho em diferentes sistemas agrícolas (itinerante e permanente), a agricultura permanente de pupunheira se mostrou mais eficiente em termos de trabalho e renda do que a agricultura itinerante. Entretanto, a prática do SAI não se dá apenas pelo seu fator produtivo, mas também, e principalmente, por aspectos culturais, históricos, de preservação do meio e da saúde humana. São suas outras funções, além das produtivas, que o tornam ainda presente nas comunidades quilombolas.

Apesar das limitações deste estudo, foi possível apontar caminhos para futuras investigações sobre a produtividade da agricultura itinerante na mata atlântica. $\mathrm{O}$ cultivo das roças é um sistema de uso da terra altamente dinâmico e complexo, que contempla uma teia de interações entre suas dimensões ecológicas, políticas, socioeconômicas e culturais. Qualquer projeto de desenvolvimento rural para estas comunidades deveria ser capaz de proporcionar a reprodução econômica e social das famílias, levando em conta a história e as particularidades dos territórios quilombolas.

\section{Agradecimentos}

Agradecemos à Fundação de Amparo à Pesquisa do Estado de São Paulo (FAPESP) às bolsas concedidas (2012/24232-5 e 2015/02001-0), que possibilitaram a execução desta pesquisa e a redação do artigo. Agradecemos ainda a Joaquim Alves da Silva Junior, Jomar Magalhães Barbosa, e às Comunidades Quilombolas São Pedro e Pedro Cubas de Cima.

\section{Referências}

Adams, C. Caiçaras na Mata Atlântica: pesquisa científica versus planejamento e gestão ambiental. São Paulo: Annablume, FAPESP, 2000a.

Adams, C. As roças e o manejo da Mata Atlântica pelos caiçaras: uma revisão. Interciencia, 25(3), 143-150, 2000 b.

Adams, C.; Munari, L. C.; Van Vliet, N.; Murrieta, R. S. S.; Piperata, B. A.; Futemma, C.; Pedroso Junior, N. N.; Taqueda, C. S.; Crevelaro, M. A.; Spressola-Prado, V. L. Diversifying incomes and losing landscape complexity in quilombola shifting cultivation communities of the Atlantic Rainforest (Brazil). Human Ecololy, 41(1), 119-137, 2013.

Andrade, A. M.; Tatto, N. (Ed.). Inventário cultural de quilombos do Vale do Ribeira. São Paulo: Câmara Brasileira do Livro, 2013.

Bayliss-Smith, T. P. The ecology of agricultural systems. Cambridge University Press, 1982.

Barbosa, J. M.; Melendez-Pastor, I.; Navarro-Pedreño, J.; Bittencourt, M. D. Remotely sensed biomass over steep slopes: an evaluation among successional stands of the Atlantic Forest, Brazil. Journal of Photogrammetry and Remote Sensing, 88, 91-100, 2014.

Barlett, P. Adaptive strategies in peasant agricultural production. Annual Review of Anthropology, 9, 545-573, 1980.

Bebbington, A. Capitals and capabilities: a framework for 
analyzing peasant viability, rural livelihoods and poverty. World Development, 27(12), 2021-2044, 1999.

Beckerman, S. Swidden in Amazonia and the Amazon Rim. In: Turner, B. L.; Brush, S. (Orgs.). Comparative farming systems. New York: The Guilford Press, p. 55-94, 1987.

Bernard, H. R. Research methods in anthropology: qualitative and quantitative approaches. Lanham: Altamira Press, 2005.

Boserup, E. Evolução agrária e pressão demográfica. São Paulo: Hucitec, Polis, 1987.

Brasil. Lei $n^{\circ} 11.428$, de 22 de dezembro de 2006. Dispõe sobre a utilização e proteção da vegetação nativa do Bioma da Mata Atlântica, e dá outras providências. Brasília: DOU 26/12/2006.

Brasil. Decreto ${ }^{\circ}$ 6.660, de 21 de novembro de 2008. Regulamenta dispositivos da Lei no 11.428, de 22 de dezembro de 2006, que dispõe sobre a utilização e proteção da vegetação nativa do Bioma Mata Atlântica. Brasília: DOU 24/11/2008.

Burger, D. M. Modelos alométricos para a estimativa da fitomassa de Mata Atlântica na Serra do Mar, SP. 2005. São Paulo, Tese (Doutorado em Ciências) - USP, 2005.

Camarano, A. A.; Abramovay, R. Êxodo rural, envelhecimento e masculinização no Brasil: panorama dos últimos cinquenta anos. Rio de Janeiro: Instituto de Pesquisa Econômica Aplicada, 1999. 28 p.

Camargo, R. A. L.; Oliveira, J. T. A. Agricultura familiar, multifuncionalidade da agricultura e ruralidade: interfaces de uma realidade complexa. Ciência Rural, 42(9), 17071714, 2012.

Carneiro, R. L. The cultivation of manioc among the Kuikuru of the Upper Xingú. In: Hames, R. B.; Vickers, W. T. (Eds). Adaptive responses of native amazonians. New York: Academic Press, 1983. p. 65-112.

Carriere, S. M.; Letourmy, P.; Mckey, D. B. Effects of remnant trees in fallows on diversity and structure of forest regrowth in a slash-and-burn agricultural system in southern Cameroon. Journal of Tropical Ecology, 18(3), 375-396, 2002.

Chayanov, A. V. On the theory of non-capitalist economic systems. In: Thorner, D.; Kerblay, B.; Smith, R. E. F. (Eds.) The theory of peasant economy. Manchester University Press,1986. p. 1-28.

Conklin, H. C. The study of shifting cultivation. Current Anthropology, 2(1), 27-61, 1961.

Cramb, R. A.; Colfer, C. J. P.; Dressler, W.; Laungaramsri, P.; Trang Le, Q.; Mulyoutami, E.; Peluso, N. L.; Wadley, R.L. Swidden transformations and rural livelihoods in Southeast Asia. Human Ecology, 37(3), 323-346, 2009.

Denevan, W. M. Campa subsistence in the Gran Pajonal of eastern Peru. The Geographical Review, 61(4), 496-518, 1971.

Dove, M. R. Theories of swidden agriculture, and the political economy of ignorance. Agroforestry Systems, 1, 85-89, 1983.

Dufumier, M. Projetos de desenvolvimento agrícola: manual para especialistas. Salvador: EDUFBA, 2. ed., 2010.

Ellis, F. Rural livelihoods and diversity in developing countries. Oxford: University Press, 2000.

FAO - Food and Agriculture Organization of the United Nations. Shifting cultivation, livelihood and food security: new and old challenges for indigenous peoples in Asia. Bangkok: FAO Press, 2015.

Fox, J.; Truong, D.M.; Rambo, T.; Tuyen, N.P.; Le Cuc, T.; Leisz, S. Shifting cultivation: a new old paradigm for managing tropical forests. Bioscience, 50(6), 521-528, 2000.

Futemma, R. T. F.; Munari, L. C.; Adams, C. The Afro-Brazilian collective land: analyzing institutional changes in the past 200 years. Latin American Research Review, 50(4), 26-48, 2015.

Gavioli, F. R.; Costa, M. B. B. As múltiplas funções da agricultura familiar: um estudo no assentamento Monte alegre, região de Araraquara (SP). Revista de Economia e Sociologia Rural, 49(2), 449-472, 2011.

Hunt, R.C. Labor productivity and agricultural development: Boserup revisited. Human Ecology, 28(2), 251-277, 2000.

Ianovali, D. A agricultura quilombola no Vale do Ribeira-SP: comparação entre as agriculturas itinerante e per- 
manente. Piracicaba, Dissertação (Mestrado em Ciências) - USP, 2015.

IEA - Instituto de Economia Agrícola. Como medir a produtividade agrícola: conceitos, métodos, e aplicações no caso de São Paulo. São Paulo, 1986.

ITESP - Fundação Instituto de Terras do Estado de São Paulo. Relatório técnico-científico sobre os remanescentes da comunidade de quilombo Sapatu/Eldorado-SP. São Paulo: Secretaria da Justiça e Defesa da Cidadania, 2000. 57 p.

ITESP - Fundação Instituto de Terras do Estado de São Paulo. Relatório técnico-científico sobre os remanescentes da comunidade de quilombo de Pedro Cubas de Cima. Município de Eldorado-SP. São Paulo: Secretaria da Justiça e Defesa da Cidadania, 2003. 43 p.

Jakovac, C. C.; Peña-Claros, M.; Kuyper, T. W.; Bongers, F. Loss of secondary-forest resilience by land-use intensification in the Amazon. Journal of Ecology, 103(1), 67-77, 2015.

Khatounian, C. A. A reconstrução ecológica da agricultura. Botucatu: Agroecológica, 2001.

Kleinman, P. J. A.; Pimentel, D.; Bryant, R.B. The ecological sustainability of slash-and-burn agriculture. Agriculture, Ecosystems \& Environment, 52(2-3), 235-249, 1995.

Luca, F. V.; Kubo, K. R. Meios de vida rurais sustentáveis em um contexto de agricultura de pousio associada à produção de carvão vegetal em comunidades rurais de Biguaçu/ SC. Desenvolvimento e Meio Ambiente, 35, 367-383, 2015.

Mazoyer, M.; Roudart, L. História das agriculturas do mundo: do neolítico à crise contemporânea. São Paulo: Editora UNESP, 2010.

Mertz, O. The relationship between length of fallow and crop yields in shifting cultivation: a rethinking. Agroforestry Systems, 55(2), 149-159, 2002.

Mertz, O.; Padoch, C.; Fox, J.; Cramb, R. A.; Leisz, S.J.; Lam, N. T.; Duc Vien, T. Swidden change in Southeast Asia: understanding causes and consequences. Human Ecology, 37(3), 259-264, 2009.

McGrath, D. G. The role of biomass in shifting cultivation. Human Ecology, 15(2), 221-242, 1987.
Munari, L. C. Memória social e ecologia histórica: a agricultura de coivara das populações quilombolas do Vale do Ribeira e sua relação com a formação da Mata Atlântica local. São Paulo, Dissertação (Mestrado em Ciências) USP, 2009.

Netting, R. M. Smallholders, householders: farm families and the ecology of intensive, sustainable agriculture. Stanford University Press, 1993.

Neves, W. A.; Ribeiro Filho, A. A.; Adams, C.; Murrieta, R. S. S.; Pedroso Junior, N. N. Coivara: cultivo itinerante na floresta tropical. Ciência Hoje, 50(297), 26-30, 2012.

Nielsen, U.; Mertz, O.; Noweg, G. T. The rationality of shifting cultivation systems: labor productivity revisited. Human Ecology, 34(2), 201-218, 2006.

Nishikawa, M. A. N.; Moro, J. R.; Bandel, G. A cultura da pupunha para produção de palmito. Piracicaba: ESALQ, 1998. 32 p. (Série Produtor Rural, 6).

Norgrove, L.; Houser, S. Estimating the consequences of fire exclusion for food crop production, soil fertility, and fallow recovery in shifting cultivation landscapes in the humid tropics. Environmental Management, 55(3), 536-549, 2015.

Padoch,C.; Pinedo-Vasquez, M. Saving slash-and-burn to save biodiversity. Biotropica,42(5), 550-552, 2010.

Pedroso Junior, N. N. Do caminho dos antigos: agricultura de corte-e-queima e intensificação agrícola em populações quilombolas do Vale do Ribeira, SP. São Paulo, Tese (Doutorado em Ciências) - USP, 2008.

Pedroso Junior, N. N.; Murrieta, R. S. S.; Adams, C. 2008. A agricultura de corte e queima: um sistema em transformação. Boletim do Museu Paraense Emílio Goeldi, 3(2), 153-174, 2008.

Queiroz, R. S. Caipiras negros no Vale do Ribeira: um estudo de antropologia econômica. São Paulo: USP, FFL$\mathrm{CH}, 1983$.

Raupp, F. M.; Beuren, I. M. Metodologia da pesquisa aplicável às ciências sociais. In: Beuren, I. M. Como elaborar trabalhos monográficos em contabilidade. 3. ed. São Paulo: Atlas, 2006. cap. 3, p. 76-97.

Ribeiro Filho, A. A. Impactos do sistema agrícola itinerante 
sobre os solos de remanescente de Mata Atlântica com uso e ocupação por comunidades quilombolas no Vale do Ribeira (São Paulo, Brasil). São Paulo, Tese (Doutorado em Ciências) - USP, 2015.

Ribeiro Filho, A. A.; Adams, C.; Manfredini, S.; Munari, L. C.; Silva Junior, J. A.; Ianovali, D.; Barbosa, J. M.; Barreiros, A. M.; Neves, W.A. Dynamics of the soil fertility in quilombola shifting cultivation communities of the Atlantic Rainforest, Brazil. Boletim do Museu Paraense Emílio Goeldi, Ciências Naturais, 13(1), 79-106, 2018.

Schwartz, N. B.; Corzo, A. R. M. Swidden counts: a Petén, Guatemala, milpa system production, carrying capacity, and sustainability in the Southern Maya Lowlands. Journal of Anthropological Research, 71(1), 69-93, 2015.

Stone, G. D. Agricultural change theory. In: Smelser, N. J.; Baltes, P. B. (Eds.) International encyclopedia of the social and behavioral sciences. Amsterdam, Elsevier, 2001. p. 329-333.

Turner, B. L; Brush, S. (Eds.) Comparative farming systems. New York: The Guilford Press, 1987.

Van Vliet, N.; Mertz, O.; Heinimann, A.; Langanke, T.; Pascual, U.; Schmook, B.; Adams, C.; Schmidt-Vogt, D.; Messerli, P.; Leisz, S.; Castella, J. C.; Jørgensen, L.; Birch-Thomsen, T.; Hett, C.; Bechbruun, T.; Ickowitz, A.; Vum, K. C.; Yasuyuki, K.; Fox, J.; Padoch, C.; Dressler, W.; Ziegler, A. D. Trends, drivers and impacts of changes in swidden cultivation in tropical forest-agriculture frontiers: a global assessment. Global Environmental Change, 22(2), 418-429, 2012.
Van Vliet, N.; Adams, C.; Vieira, I. C. G.; Mertz;O. "Slash and burn" and "shifting" cultivation systems in forest agriculture frontiers from the Brazilian Amazon. Society and Natural Resources, 26(12), 1454-1467, 2013.

Vicente, N. R.; Fantini, A. C. Transformações no sistema tradicional de roça itinerante na Mata Atlântica do litoral sul brasileiro. Interthesis, 11(2), 183-203, 2014.

Vickers, W. T. Tropical forest mimicry in swiddens: a reassessment of Geertz's model with Amazonian data. Human Ecology, 11(1), 35-45, 1983.

Whitmore, T. C. An introduction to tropical rain forests. New York: Oxford University Press, 1998.

Wood, S. L. R.; Rhemtulla, J. M.; Coomes, O. T. Intensification of tropical fallow-based agriculture: trading-off ecosystem services for economic gain in shifting cultivation landscapes? Agriculture, Ecosystems \& Environment, 215(1), 47-56, 2016. 